\title{
EIGENFUNCTION BEHAVIOR AND ADAPTIVE FINITE ELEMENT APPROXIMATIONS OF NONLINEAR EIGENVALUE PROBLEMS IN QUANTUM PHYSICS
}

\author{
BIN YANG ${ }^{1,2}$ AND Ainui ZHOU ${ }^{1,2, *}$
}

\begin{abstract}
In this paper, we investigate a class of nonlinear eigenvalue problems resulting from quantum physics. We first prove that for any open set $G$, there exists an eigenfunction that cannot be a polynomial on $G$, which may be reviewed as a refinement of the classic unique continuation property. Then we apply the non-polynomial behavior of the eigenfunction to show that the adaptive finite element approximations are convergent even if the initial mesh is not fine enough. We finally remark that similar arguments can be applied to a class of linear eigenvalue problems that improve the relevant existing results.
\end{abstract}

Mathematics Subject Classification. 35Q55, 65N15, 65N25, 65N30, 81Q05.

Received July 30, 2019. Accepted November 10, 2020.

\section{INTRODUCTION}

In this paper, we investigate the eigenfunction behavior and adaptive finite element approximations of the following nonlinear eigenvalue problem: find $\left(\lambda_{i}, \phi_{i}\right) \in \mathbb{R} \times H_{0}^{1}(\Omega)$ such that $\int_{\Omega} \phi_{i} \phi_{j}=\delta_{i j}(i, j=1,2, \ldots, N)$ and

$$
(-\kappa \Delta+V+\mathcal{N}(\rho)) \phi_{i}=\lambda_{i} \phi_{i}, \quad \text { in } \Omega, \quad i=1,2, \ldots, N,
$$

where $\Omega \subset \mathbb{R}^{3}, N \geq 1, \kappa>0, \rho=\sum_{i=1}^{N}\left|\phi_{i}\right|^{2}, V: \Omega \rightarrow \mathbb{R}$ is a given function, and $\mathcal{N}$ maps a nonnegative function to some function on $\Omega$. We observe that the Schrödinger-Newton equation modeling the quantum state reduction [18,25], the Gross-Pitaevskii equation (GPE) describing Bose-Einstein condensates (BEC) [2,35], and the Thomas-Fermi-von Weizsäcker (TFvW) type equations and the Kohn-Sham equations appearing in the electronic density functional theory [3, 9, 20-22] are typical examples of (1.1).

We understand that it is significant to solve (1.1) accurately and efficiently. And we note that the a priori knowledge of their eigenfunctions is very helpful in the design and analysis of numerical methods. To improve the approximation accuracy and reduce the computational cost in solving the eigenvalue problem, we see from the regularity behavior of eigenfunctions $[17,34]$ that adaptive finite element approaches should be employed

\footnotetext{
Keywords and phrases. Adaptive finite element approximation, complexity, convergence, nonlinear eigenvalue problem, non-polynomial behavior, unique continuation property.

1 LSEC, Institute of Computational Mathematics and Scientific/Engineering Computing, Academy of Mathematics and Systems Science, Chinese Academy of Sciences, Beijing 100190, P.R. China.

2 School of Mathematical Sciences, University of Chinese Academy of Sciences, Beijing 100049, P.R. China.

*Corresponding author: azhou@lsec.cc.ac.cn
} 
(see also [7,10-14,24,31] and references cited therein). We observe that the adaptive finite element analysis of the nonlinear eigenvalue problem $(1.1)$ in $[7,8,10]$ requires that the initial mesh size is small enough. However, our numerical experiments show that the small initial mesh size requirement is unnecessary $[7,8,10]$. In this paper, we study the adaptive finite element approximations when the initial mesh is not fine, for which we need to apply an eigenfunction behavior that is also investigated.

We see that the unique continuation property is significant in the context of partial differential equations (see, e.g., $[19,28,33]$ and references cited therein). After looking into the eigenfunction behavior of (1.1), we find that for any open set $G$, there exists an eigenfunction that cannot be a polynomial on $G$, which may be reviewed as a refinement of the classic unique continuation property and is indeed a key in our adaptive finite element analysis. Taking into account the eigenfunction behavior, we are indeed able to prove the convergence of adaptive finite element approximations without the requirement of the small initial mesh size.

The rest of this paper is organized as follows. In the next section, we describe some basic notation and review the adaptive finite element method for solving (1.1). Then we show some polynomial properties which are crucial in our adaptive finite element analysis. In Section 3, we obtain that for any open set $G$ of $\Omega$, there exists an eigenfunction of (1.1) that cannot be a polynomial on $G$ under some assumptions, which may be reviewed as an extension and refinement of the classic unique continuation property. In Section 4, based on the non-polynomial behavior of eigenfunctions, we study the convergence of the adaptive finite element method. We finally remark that similar arguments can be applied to a class of linear eigenvalue problems that improve the relevant existing results.

\section{Preliminaries}

Let $\Omega \subset \mathbb{R}^{3}$ be a convex polyhedral bounded domain. We will always use $G$ for a nonempty open set of $\mathbb{R}^{d}(d \geq 1)$ throughout this paper. Let $\mathbb{Q}_{+}=\mathbb{Q} \cap[0, \infty)$ and $\alpha=\left(\alpha_{1}, \alpha_{2}, \ldots, \alpha_{d}\right) \in \mathbb{Q}_{+}^{d}$ be a $d$-tuple. We denote $|\alpha|=\alpha_{1}+\alpha_{2}+\ldots+\alpha_{d}$, define $x^{\alpha}=\xi_{1}^{\alpha_{1}} \xi_{2}^{\alpha_{2}} \xi_{3}^{\alpha_{3}}$ for any $x=\left(\xi_{1}, \xi_{2}, \xi_{3}\right) \in \mathbb{R}^{3}$ and $\alpha=\left(\alpha_{1}, \alpha_{2}, \alpha_{3}\right) \in \mathbb{Q}_{+}^{3}$, and use the notation

$$
\partial_{i} \phi=\frac{\partial \phi}{\partial \xi_{i}}, i=1,2,3 .
$$

For any $\alpha=\left(\alpha_{1}, \alpha_{2}, \ldots, \alpha_{d}\right), \beta=\left(\beta_{1}, \beta_{2}, \ldots, \beta_{d}\right) \in \mathbb{Q}_{+}^{d}$, we denote $\alpha \succ \beta$ if the first non-zero element of $\alpha-\beta=\left(\alpha_{1}-\beta_{1}, \alpha_{2}-\beta_{2}, \ldots, \alpha_{d}-\beta_{d}\right)$ is larger than 0 and $\alpha \succcurlyeq \beta$ if $\alpha \succ \beta$ or $\alpha=\beta$. For convenience, we define

$$
\mathcal{P}_{\mathbb{Q}_{+}}(G)=\left\{\sum_{\alpha \in I \subset \mathbb{Q}_{+}^{d}} a_{\alpha} x^{\alpha}: a_{\alpha}, x^{\alpha} \in \mathbb{R}, \forall \alpha \in I, \forall x \in G,|I|<\infty\right\},
$$

where $|I|$ means the cardinality of $I$. We shall use the notation

$$
\mathcal{P}_{\mathbb{Q}_{+}}^{\mu}(G)=\left\{p^{\mu}: p \in \mathcal{P}_{\mathbb{Q}_{+}}(G) \text { and } p^{\mu}(x) \in \mathbb{R} \forall x \in G\right\}
$$

for any $\mu \geq 0$. We call $a_{\alpha} x^{\alpha}$ with $a_{\alpha} \neq 0$ a monomial. Denote $|\alpha|$ the degree of the monomial $a_{\alpha} x^{\alpha}$. We shall let the degree of the polynomial 0 be $-\infty$. For any $p \in \mathcal{P}_{\mathbb{Q}_{+}}(G)$, define $\operatorname{deg} p$ as the max degree of terms of $p$, which is called the degree of $p$. We shall also denote $\operatorname{deg} p^{\mu}=\mu \operatorname{deg} p$ for any $p \in \mathcal{P}_{\mathbb{Q}_{+}}(G)$ and $\mu \in \mathbb{Q}_{+}$and $\operatorname{deg}(p / q)=\operatorname{deg} p-\operatorname{deg} q$ for any $p, q \in \mathcal{P}_{\mathbb{Q}_{+}}(G)$. Let $\mathcal{P}_{\ell}(G)$ be the set of real polynomials on $G$ with a degree not larger than $\ell$. It is clear that $\mathcal{P}_{\ell}(G) \subset \mathcal{P}_{\mathbb{Q}_{+}}(G)$. The standard notation $W^{s, p}(\Omega)(s \geq 0)$ for Sobolev spaces and their associated norms $\|\cdot\|_{s, p, \Omega}$ shall also be used [1]. We write

$$
G \subset \subset \Omega
$$

if $\bar{G} \subset \Omega$ and $\bar{G}$ is compact. $W_{\text {loc }}^{s, p}(\Omega)$ denotes the space of the function $v$ satisfying that for any open set $G \subset \subset \Omega$, $v \in W^{s, p}(G)$. For $p=2$, we denote $H^{s}(\Omega)=W^{s, 2}(\Omega), H_{\mathrm{loc}}^{s}(\Omega)=W_{\mathrm{loc}}^{s, 2}(\Omega), H_{0}^{1}(\Omega)=\left\{v \in H^{1}(\Omega):\left.v\right|_{\partial \Omega}=0\right\}$, 
where $\left.v\right|_{\partial \Omega}$ is understood in the sense of trace, $\|\cdot\|_{s, \Omega}=\|\cdot\|_{s, 2, \Omega}$. We use $\mathscr{P}\left(s,\left(c_{1}, c_{2}\right)\right)$ to denote a class of functions satisfying some growth conditions:

$$
\mathscr{P}\left(s,\left(c_{1}, c_{2}\right)\right)=\left\{f: \exists a_{1}, a_{2} \in \mathbb{R} \text { such that } c_{1} t^{s}+a_{1} \leq f(t) \leq c_{2} t^{s}+a_{2} \quad \forall t \geq 0\right\}
$$

with $c_{1} \in \mathbb{R}$ and $c_{2}, s \in[0, \infty)$.

\subsection{Quantum eigenvalue problem}

We consider the nonlinear eigenvalue problem (1.1) where $V$ is of the form

$$
V=-\sum_{j=1}^{M} \frac{f_{j}}{g_{j}}
$$

with $f_{j}, g_{j} \in \mathcal{P}_{\mathbb{Q}_{+}}^{\mu}\left(\mathbb{R}^{3}\right)(j=1,2, \ldots, M)$ for some $\mu \in \mathbb{Q}_{+}$. Assume that $\mathcal{N}$ is divided into two parts:

$$
\mathcal{N}(\rho)=\mathcal{N}_{1}(\rho)+\mathcal{N}_{2}(\rho)
$$

where $\rho=\sum_{i=1}^{N}\left|\phi_{i}\right|^{2}$. Let $\mathcal{N}_{1}:[0, \infty) \rightarrow \mathbb{R}$ be defined by

$$
\mathcal{N}_{1}(t)=\sum_{i=1}^{K} \frac{p_{i}(t)}{h_{i}(t)} \ln q_{i}(t)
$$

with $p_{i}, h_{i} \in \mathcal{P}_{\mathbb{Q}_{+}}([0, \infty))$, where $q_{i}$ are polynomials satisfying $\left.q_{i}\right|_{[0, \infty)}>0$ and $q_{i} \neq 1(i=1,2, \cdots, K)$, and $\mathcal{N}_{2}$ be given by a convolution integral

$$
\mathcal{N}_{2}(\rho)=\alpha \int_{\Omega} \frac{\rho(y)}{|\cdot-y|} \mathrm{d} y
$$

with some constant $\alpha$.

The energy functional associated with (1.1) is

$$
E(\Phi)=\int_{\Omega}\left(\kappa \sum_{i=1}^{N}\left|\nabla \phi_{i}\right|^{2}+V \rho_{\Phi}+\mathcal{E}\left(\rho_{\Phi}\right)\right)+\frac{\alpha}{2} D\left(\rho_{\Phi}, \rho_{\Phi}\right)
$$

for $\Phi=\left(\phi_{1}, \phi_{2}, \ldots, \phi_{N}\right) \in \mathcal{H} \equiv\left(H_{0}^{1}(\Omega)\right)^{N}$, where $\rho_{\Phi}=\sum_{i=1}^{N}\left|\phi_{i}\right|^{2}, \mathcal{E}:[0, \infty) \rightarrow \mathbb{R}$ is defined by

$$
\mathcal{E}(s)=\int_{0}^{s} \mathcal{N}_{1}(t) \mathrm{d} t
$$

and $D(\cdot, \cdot)$ is a bilinear form as follows

$$
D(f, g)=\int_{\Omega} \int_{\Omega} \frac{f(x) g(y)}{|x-y|} \mathrm{d} x \mathrm{~d} y .
$$

For any $\Phi \in \mathcal{H}$, we denote

$$
\|\Phi\|_{s, \Omega}=\left(\sum_{i=1}^{N}\left\|\phi_{i}\right\|_{s, \Omega}^{2}\right)^{1 / 2} .
$$

We see that (1.1) includes the GPE, the Schrödinger-Newton equation, the TFvW type equation, and the Kohn-Sham equation (see Rem. 3.7, Examples 3.9, 3.10, and 3.11 for more details).

Let $\mathcal{Q}$ be a subset of $\mathcal{H}$ :

$$
\mathcal{Q}=\left\{\Phi \in \mathcal{H}: \Phi^{T} \Phi=I^{N \times N}\right\}
$$


where $\Phi^{T} \Psi=\left(\int_{\Omega} \phi_{i} \psi_{j}\right) \in \mathbb{R}^{N \times N}$. The ground state charge density of (1.1) is obtained by solving the minimization problem

$$
\inf \{E(\Phi): \Phi \in \mathcal{Q}\} .
$$

We see that any minimizer $\Phi=\left(\phi_{1}, \ldots, \phi_{N}\right)$ of $(2.5)$ satisfies

$$
\left\{\begin{aligned}
\left\langle H_{\Phi} \phi_{i}, v\right\rangle & =\left(\sum_{j=1}^{N} \lambda_{j i} \phi_{j}, v\right) \quad \forall v \in H_{0}^{1}(\Omega), \quad i=1,2, \ldots, N \\
\int_{\Omega} \phi_{i} \phi_{j} & =\delta_{i j}
\end{aligned}\right.
$$

where $H_{\Phi}: H_{0}^{1}(\Omega) \rightarrow H^{-1}(\Omega)$ is the Hamiltonian operator defined by

$$
\left\langle H_{\Phi} u, v\right\rangle=\kappa(\nabla u, \nabla v)+\left(V \rho_{\Phi}+\mathcal{N}\left(\rho_{\Phi}\right) u, v\right) \quad \forall u, v \in H_{0}^{1}(\Omega)
$$

and

$$
\Lambda=\left(\lambda_{i j}\right)_{i, j=1}^{N}=\left(\int_{\Omega} \phi_{i} H_{\Phi} \phi_{j}\right)_{i, j=1}^{N}
$$

is the Lagrange multiplier. We call $(\Lambda, \Phi)$ a state of $(2.6)$ and define the set of ground states by

$$
\Theta=\left\{(\Lambda, \Phi) \in \mathbb{R}^{N \times N} \times \mathcal{Q}: E(\Phi)=\min _{\Psi \in \mathcal{Q}} E(\Psi) \text { and }(\Lambda, \Phi) \text { solves }(2.6)\right\} .
$$

We define the set of states of (2.6) by

$$
\mathcal{W}=\left\{(\Lambda, \Phi) \in \mathbb{R}^{N \times N} \times \mathcal{H}:(\Lambda, \Phi) \text { solves }(2.6)\right\} .
$$

Since the electron density $\rho_{\Phi}$ and the operator $H_{\Phi}$ are invariant under any unitary transform, we may diagonalize the Lagrange multiplier $\Lambda$ and arrive at

$$
\left\{\begin{aligned}
\left\langle H_{\Phi} \phi_{i}, v\right\rangle & =\lambda_{i}\left(\phi_{i}, v\right) \quad \forall v \in H_{0}^{1}(\Omega), \quad i=1,2, \ldots, N, \\
\int_{\Omega} \phi_{i} \phi_{j} & =\delta_{i j},
\end{aligned}\right.
$$

which is equivalent to (2.6) and a weak form of (1.1).

In practice, the ground states are usually approximated by solving the lowest $N$ eigenpairs of (2.7) [22].

\subsection{An adaptive finite element method}

Let $d_{\Omega}$ be the diameter of $\Omega$ and $\left\{\mathcal{T}_{h}\right\}$ be a shape regular family of nested conforming meshes over $\Omega$ with size $h \in\left(0, d_{\Omega}\right)$ : there exists a constant $\gamma^{*}$ such that

$$
\frac{h_{\tau}}{\rho_{\tau}} \leq \gamma^{*} \quad \forall \tau \in \mathcal{T}_{h},
$$

where $h_{\tau}$ is the diameter of $\tau, \rho_{\tau}$ is the diameter of the biggest ball contained in $\tau$, and $h=\max \left\{h_{\tau}: \tau \in \mathcal{T}_{h}\right\}$. Let $\mathcal{E}_{h}$ denote the set of interior faces of $\mathcal{T}_{h}$. We shall also use a slight abuse of notation that $h$ denotes the mesh size function defined by

$$
h(x)=h_{\tau}, \quad x \in \tau \quad \forall \tau \in \mathcal{T}_{h} .
$$

Let $S^{h}(\Omega) \subset H^{1}(\Omega)$ be the corresponding finite element space consisting of continuous piecewise polynomials over $\mathcal{T}_{h}$ of degrees not larger than $n \geq 1$ and

$$
S_{0}^{h}(\Omega)=S^{h} \cap H_{0}^{1}(\Omega) .
$$


Let $V_{h}=\left(S_{0}^{h}(\Omega)\right)^{N}$.

Consider the finite element approximation of (2.5):

$$
\inf \left\{E\left(\Phi_{h}\right): \Phi_{h} \in V_{h} \cap \mathcal{Q}\right\}
$$

We see that any minimizer $\Phi_{h}=\left(\phi_{1, h}, \ldots, \phi_{N, h}\right)$ of $(2.8)$ solves the Euler-Lagrange equation

$$
\left\{\begin{aligned}
\left\langle H_{\Phi_{h}} \phi_{i, h}, v\right\rangle & =\left(\sum_{j=1}^{N} \lambda_{j i, h} \phi_{j, h}, v\right) \quad \forall v \in S_{0}^{h}(\Omega), \quad i=1,2, \ldots, N \\
\int_{\Omega} \phi_{i, h} \phi_{j, h} & =\delta_{i j}
\end{aligned}\right.
$$

with the Lagrange multiplier

$$
\Lambda_{h}=\left(\lambda_{i j, h}\right)_{i, j=1}^{N}=\left(\int_{\Omega} \phi_{i, h} H_{\Phi_{h}} \phi_{j, h}\right)_{i, j=1}^{N}
$$

when the energy functional is differentiable. Define the set of finite dimensional ground state solutions:

$$
\Theta_{h}=\left\{\left(\Lambda_{h}, \Phi_{h}\right) \in \mathbb{R}^{N \times N} \times\left(\mathcal{Q} \cap V_{h}\right): E\left(\Phi_{h}\right)=\min _{\Psi \in \mathcal{Q} \cap V_{h}} E(\Psi),\left(\Lambda_{h}, \Phi_{h}\right) \text { solves }(2.9)\right\} .
$$

Using a unitary transformation, we have the following discrete equation

$$
\left\{\begin{aligned}
\left\langle H_{\Phi_{h}} \phi_{i, h}, v\right\rangle & =\lambda_{i, h}\left(\phi_{i, h}, v\right) \quad \forall v \in H_{0}^{1}(\Omega), \quad i=1,2, \ldots, N, \\
\int_{\Omega} \phi_{i, h} \phi_{j, h} & =\delta_{i j} .
\end{aligned}\right.
$$

In practice, we usually solve and obtain the lowest $N$ eigenpairs of (2.10) [22].

We recall that the adaptive finite element method is to repeat the following procedure [10]:

$$
\text { Solve } \rightarrow \text { Estimate } \rightarrow \text { Mark } \rightarrow \text { Refine. }
$$

For convenience, we shall replace subscript $h\left(\right.$ or $h_{k}$ ) by an iteration counter $k$ of the adaptive method afterwards.

Given an initial triangulation $\mathcal{T}_{0}$ so that the dimension of $S_{0}^{h}$ is larger than or equal to $N$, the above procedure generates a sequence of nested triangulations $\mathcal{T}_{k}(k=1,2, \ldots)$. Given an iteration counter $k$, the procedure "Solve" is to get the discrete ground state solution of (2.9) over $\mathcal{T}_{k}$. The procedure "Estimate" determines the element indicators for all elements $\tau \in \mathcal{T}_{k}$. In this step, a posteriori error estimators play a critical role. Then, the element indicators are used by the procedure "Mark" to create a subset $\mathcal{M}_{k}$ of marked elements $\tau \in \mathcal{T}_{k}$. Finally, the procedure "Refine" produces a new partition $\mathcal{T}_{k+1}$ by refining all elements in $\mathcal{M}_{k}$ at least once. Here we use a shape-regular bisection for the refinement. To keep the conformity, we usually partition a few more elements $\tau \in \mathcal{T}_{k} \backslash \mathcal{M}_{k}$.

Given a triangulation $\mathcal{T}_{h}$ and the corresponding finite element solution $\left(\Lambda_{h}, \Phi_{h}\right)$, we respectively define the element residual $\mathcal{R}_{\tau}\left(\Phi_{h}\right)$ and the jump $J_{e}\left(\Phi_{h}\right)$ by

$$
\begin{aligned}
\mathcal{R}_{\tau}\left(\Phi_{h}\right) & =\left(H_{\Phi_{h}} \phi_{i, h}-\sum_{j=1}^{N} \lambda_{j i, h} \phi_{j, h}\right)_{i=1}^{N} \text { in } \tau \in \mathcal{T}_{h}, \\
J_{e}\left(\Phi_{h}\right) & =\left(j_{e}\left(\phi_{i, h}\right)\right)_{i=1}^{N}, \quad j_{e}\left(\phi_{i, h}\right)=\left.\kappa \nabla \phi_{i, h}\right|_{\tau_{1}} \cdot \overrightarrow{n_{1}}+\left.\kappa \nabla \phi_{i, h}\right|_{\tau_{2}} \cdot \overrightarrow{n_{2}}
\end{aligned}
$$


where $e$ is the common face of elements $\tau_{1}$ and $\tau_{2}$ with unit outward normals $\overrightarrow{n_{1}}$ and $\overrightarrow{n_{2}}$. For $\tau \in \mathcal{T}_{h}$, we define the local error indicator $\eta_{h}\left(\Phi_{h}, \tau\right)$ as follows:

$$
\eta_{h}^{2}\left(\Phi_{h}, \tau\right)=h_{\tau}^{2}\left\|\mathcal{R}_{\tau}\left(\Phi_{h}\right)\right\|_{0, \tau}^{2}+\sum_{e \in \mathcal{E}_{h}, e \subset \partial \tau} h_{e}\left\|J_{e}\left(\Phi_{h}\right)\right\|_{0, e}^{2}
$$

Depending on the a posteriori error indicators $\left\{\eta_{k}\left(\Phi_{k}, \tau\right)\right\}_{\tau \in \mathcal{T}_{k}}$, the procedure "Mark" gives a strategy to create a subset of elements $\mathcal{M}_{k}$ of $\mathcal{T}_{k}$. Here, we consider "maximum strategy" which only requires that the set of marked elements $\mathcal{M}_{k}$ contains at least one element of $\mathcal{T}_{k}$ holding the largest value estimator. Namely, there exists at least one element $\tau_{k}^{\max } \in \mathcal{M}_{k}$ such that

$$
\eta_{k}\left(\Phi_{k}, \tau_{k}^{\max }\right)=\max _{\tau \in \mathcal{T}_{k}} \eta_{k}\left(\Phi_{k}, \tau\right)
$$

The adaptive finite element algorithm for solving (2.7) is stated as follows $[7,8,10]$ :

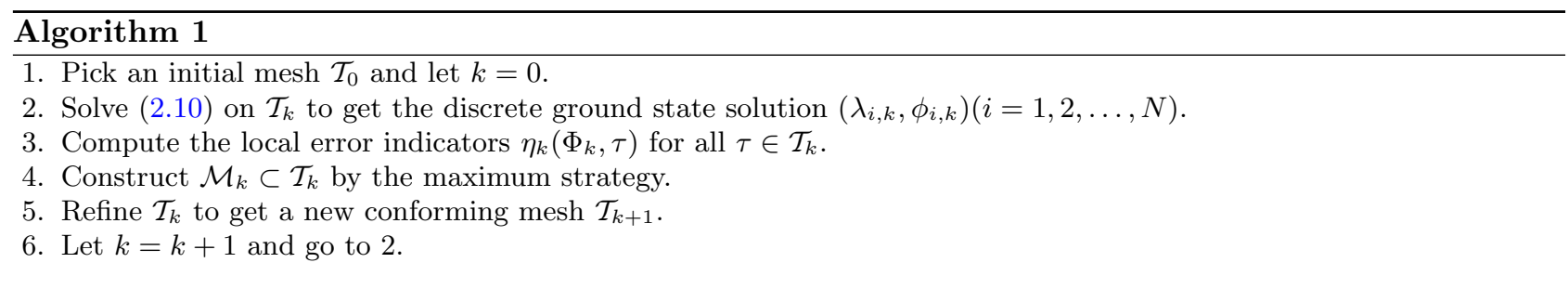

We observe that there are a number of works on analyzing adaptive finite element methods in the literature. We refer to $[4-6,11,15,16]$ and references cited therein for linear eigenvalue problems and to $[7,8,10]$ for nonlinear cases when the initial mesh is fine enough. We see that under the so-called Non-Degeneracy assumption ${ }^{1},[14]$ proved the convergence of an adaptive finite element method starting from any initial mesh for some linear elliptic eigenvalue problems.

\subsection{A polynomial theory}

In our analysis, we need the following basic results, which are motivated by [36].

Lemma 2.1. Let $k$ be a prime number. Then there exist polynomials with respect to $t_{1}, t_{2}, \ldots, t_{k}$

$$
\left\{p_{j}\left(t_{1}, t_{2}, \ldots, t_{k}\right): j=2, \ldots, k\right\}
$$

with real coefficients satisfying

(1) $p_{j}\left(t_{1}, t_{2}, \ldots, t_{k}\right)$ is a polynomial of a degree not larger than $j-1$ with respect to $t_{k}$ and

$$
p_{j}\left(\lambda t_{1}, \lambda t_{2}, \ldots, \lambda t_{k-1}, t_{k}\right)=\lambda^{j} p_{j}\left(t_{1}, t_{2}, \ldots, t_{k-1}, t_{k}\right) \forall \lambda \in \mathbb{R}
$$

(2) if $\gamma, a_{1}, a_{2}, \ldots, a_{k-1}, t \in \mathbb{R}$ satisfy $\gamma=a_{1} t+a_{2} t^{2}+\cdots+a_{k-1} t^{k-1}$, then

$$
\gamma^{k}+\sum_{j=2}^{k} p_{j}\left(a_{1}, a_{2}, \ldots, a_{k-1}, t^{k}\right) \gamma^{k-j}=0 .
$$

\footnotetext{
${ }^{1}$ No eigenfunction is equal to a polynomial of degree $\leq n$ on an open subset of $\Omega$, where $n$ denotes the polynomial degree of the finite element bases being used.
} 
The proof of Lemma 2.1 is given in Appendix A.

Lemma 2.2. Suppose $k$ is a prime. Then for any positive integer $n$, there exist polynomials

$$
\left\{H_{n, j}\left(t_{1}, t_{2}, \ldots, t_{n}\right): j=0,1,2, \ldots, k^{n-1}\right\}
$$

with real coefficients satisfying

(1) $H_{n, 0}\left(t_{1}, t_{2}, \ldots, t_{n}\right)=1, H_{n, j}\left(t_{1}, t_{2}, \ldots, t_{n}\right)\left(j=1,2, \ldots, k^{n-1}\right)$ are homogeneous:

$$
H_{n, j}\left(\lambda t_{1}, \lambda t_{2}, \ldots, \lambda t_{n}\right)=\lambda^{j} H_{n, j}\left(t_{1}, t_{2}, \ldots, t_{n}\right) \forall \lambda \in \mathbb{R},
$$

and $(-1)^{k^{n-1}} H_{n, k^{n-1}}\left(t_{1}, t_{2}, \ldots, t_{n}\right)$ is a monic polynomial of a degree $k^{n-1}$ with respect to each variable $t_{l}(l=1,2, \ldots, n)$;

(2) if $\gamma, t_{1}, t_{2}, \ldots, t_{n} \in \mathbb{R}$ satisfy $\gamma=\sum_{j=1}^{n} t_{j}$, then

$$
\sum_{j=0}^{k^{n-1}} H_{n, j}\left(t_{1}^{k}, t_{2}^{k}, \ldots, t_{n}^{k}\right) \gamma^{k\left(k^{n-1}-j\right)}=0 .
$$

Proof. We prove the conclusion by induction on $n$. First, for $n=1, \gamma=t_{1}$ implies $\gamma^{k}-t_{1}^{k}=0$, which shows that Lemma 2.2 is true when $n=1$, namely, $H_{1,1}\left(t_{1}\right)=-t_{1}$. Assume that Lemma 2.2 is true for $n \geq 1$. We show that Lemma 2.2 is true for $n+1$. Let

$$
\sum_{j=1}^{n+1} t_{j}=\gamma
$$

It follows from the induction hypothesis and

$$
\sum_{j=1}^{n} t_{j}=\gamma-t_{n+1}
$$

that there exist polynomials

$$
\left\{H_{n, j}\left(s_{1}, s_{2}, \ldots, s_{n}\right): j=0,1,2, \ldots, k^{n-1}\right\}
$$

with real coefficients satisfying that $(-1)^{k^{n-1}} H_{n, k^{n-1}}\left(s_{1}, s_{2}, \ldots, s_{n}\right)$ is a monic polynomial of a degree $k^{n-1}$ with respect to each variable $s_{l}(l=1,2, \ldots, n), H_{n, j}\left(s_{1}, s_{2}, \ldots, s_{n}\right)\left(j=1,2, \ldots, k^{n-1}\right)$ are homogeneous, and

$$
\sum_{j=0}^{k^{n-1}} H_{n, j}\left(t_{1}^{k}, t_{2}^{k}, \ldots, t_{n}^{k}\right)\left(\gamma-t_{n+1}\right)^{k\left(k^{n-1}-j\right)}=0 .
$$

We obtain from Newton binomial theory that

$$
\sum_{i=1}^{k-1} a_{i} \gamma^{k}\left(-\frac{t_{n+1}}{\gamma}\right)^{i}=a,
$$

where

$$
\begin{aligned}
a= & H_{n, k^{n-1}}\left(t_{1}^{k}, t_{2}^{k}, \ldots, t_{n}^{k}\right)+\left(-t_{n+1}\right)^{k^{n}}+\gamma^{k^{n}}+\sum_{\ell=1}^{k^{n-1}-1}\left(\begin{array}{c}
k^{n} \\
k \ell
\end{array}\right) \gamma^{k^{n}-k \ell}\left(-t_{n+1}\right)^{k \ell} \\
& +\sum_{j=1}^{k^{n-1}-1} H_{n, j}\left(t_{1}^{k}, t_{2}^{k}, \ldots, t_{n}^{k}\right) \sum_{\ell=0}^{k^{n-1}-j}\left(\begin{array}{c}
k^{n}-k j \\
k \ell
\end{array}\right) \gamma^{k^{n}-k j-k \ell}\left(-t_{n+1}\right)^{k \ell}, \\
a_{i}= & -\sum_{j=0}^{k^{n-1}-1} H_{n, j}\left(t_{1}^{k}, t_{2}^{k}, \ldots, t_{n}^{k}\right) \sum_{\ell=1}^{k^{n-1}-j}\left(\begin{array}{c}
k^{n}-k j \\
k \ell-k+i
\end{array}\right) \gamma^{k^{n}-k j-k \ell}\left(-t_{n+1}\right)^{k \ell-k}, \\
& i=1,2, \ldots, k-1 .
\end{aligned}
$$


Let polynomials $P$ and $P_{i}(i=1,2, \ldots, k-1)$ be

$$
\begin{aligned}
P\left(s_{1}, s_{2}, \ldots, s_{n+2}\right)= & H_{n, k^{n-1}}\left(s_{1}, s_{2}, \ldots, s_{n}\right)+\left(-s_{n+1}\right)^{k^{n-1}}+s_{n+2}^{k^{n-1}}+\sum_{\ell=1}^{k^{n-1}-1}\left(\begin{array}{c}
k^{n} \\
k \ell
\end{array}\right) s_{n+2}^{k^{n-1}-\ell}\left(-s_{n+1}\right)^{\ell} \\
& +\sum_{j=1}^{k^{n-1}-1} H_{n, j}\left(s_{1}, s_{2}, \ldots, s_{n}\right) \sum_{\ell=0}^{k^{n-1}-j}\left(\begin{array}{c}
k^{n}-k j \\
k \ell
\end{array}\right) s_{n+2}^{k^{n-1}-j-\ell}\left(-s_{n+1}\right)^{\ell}, \\
P_{i}\left(s_{1}, s_{2}, \ldots, s_{n+2}\right)= & -\sum_{j=0}^{k^{n-1}-1} H_{n, j}\left(s_{1}, s_{2}, \ldots, s_{n}\right) \sum_{\ell=1}^{k^{n-1}-j}\left(\begin{array}{c}
k^{n}-k j \\
k \ell-k+i
\end{array}\right) s_{n+2}^{k^{n-1}-j-\ell}\left(-s_{n+1}\right)^{\ell-1} \\
& i=1,2, \ldots, k-1 .
\end{aligned}
$$

It is clear that $P$ and $P_{i}(i=1,2, \ldots, k-1)$ are homogeneous, $\operatorname{deg} P=k^{n-1}, \operatorname{deg} P_{i}=k^{n-1}-1(i=1,2, \ldots, k-1)$, and

$$
\begin{aligned}
a & =P\left(t_{1}^{k}, t_{2}^{k}, \ldots, t_{n+1}^{k}, \gamma^{k}\right), \\
a_{i} & =P_{i}\left(t_{1}^{k}, t_{2}^{k}, \ldots, t_{n+1}^{k}, \gamma^{k}\right) \quad i=1,2, \ldots, k-1 .
\end{aligned}
$$

Since $k$ is a prime, we see from $(2.11)$ that there exist $\left\{p_{j}\left(t_{1}, t_{2}, \ldots, t_{k}\right): j=2,3, \ldots, k\right\}$ satisfying Lemma 2.1, namely,

or

$$
0=a^{k}+\sum_{j=2}^{k} p_{j}\left(a_{1} \gamma^{k}, a_{2} \gamma^{k}, \ldots, a_{k-1} \gamma^{k},\left(-t_{n+1}\right)^{k} / \gamma^{k}\right) a^{k-j},
$$

$$
0=a^{k}+\sum_{j=2}^{k} p_{j}\left(a_{1}, a_{2}, \ldots, a_{k-1},\left(-t_{n+1}\right)^{k} / \gamma^{k}\right) \gamma^{k j} a^{k-j} .
$$

We define $Q\left(s_{1}, s_{2}, \ldots, s_{n+2}\right)$ by

$$
Q=P^{k}+\sum_{j=2}^{k} p_{j}\left(P_{1}, P_{2}, \ldots, P_{k-1},(-1)^{k} s_{n+1} / s_{n+2}\right) s_{n+2}^{j} P^{k-j} .
$$

Thus it follows from (1) in Lemma 2.1 and (2.12), (2.13) that $Q$ is a homogeneous polynomial of a degree $k^{n}$ and

$$
a^{k}+\sum_{j=2}^{k} p_{j}\left(a_{1}, a_{2}, \ldots, a_{k-1},\left(-t_{n+1}\right)^{k} / \gamma^{k}\right) \gamma^{k j} a^{k-j}=Q\left(t_{1}^{k}, t_{2}^{k}, \ldots, t_{n+1}^{k}, \gamma^{k}\right) .
$$

We see that $Q$ can be expressed as

$$
Q\left(s_{1}, s_{2}, \ldots, s_{n+2}\right)=\sum_{j=0}^{k^{n}} H_{n+1, j}\left(s_{1}, s_{2}, \ldots, s_{n+1}\right) s_{n+2}^{k^{n}-j},
$$

where $H_{n+1, j}\left(j=1,2, \ldots, k^{n}\right)$ are homogeneous:

$$
H_{n+1, j}\left(\lambda s_{1}, \lambda s_{2}, \ldots, \lambda s_{n+1}\right)=\lambda^{j} H_{n+1, j}\left(s_{1}, s_{2}, \ldots, s_{n+1}\right) \forall \lambda \in \mathbb{R} .
$$

It is not difficult to see that $H_{n+1,0}\left(s_{1}, s_{2}, \ldots, s_{n}\right)=1$. We obtain from the definitions of $P, P_{i}$ and $Q$ that

$$
\begin{aligned}
& H_{n+1, k^{n}}\left(s_{1}, s_{2}, \ldots, s_{n+1}\right) \\
& \quad=\left(H_{n, k^{n-1}}\left(s_{1}, s_{2}, \ldots, s_{n}\right)+\left(-s_{n+1}\right)^{k^{n-1}}+\sum_{j=1}^{k^{n-1}-1} H_{n, j}\left(s_{1}, s_{2}, \ldots, s_{n}\right)\left(-s_{n+1}\right)^{k^{n-1}-j}\right)^{k} .
\end{aligned}
$$


Since $(-1)^{k^{n-1}} H_{n, k^{n-1}}\left(s_{1}, s_{2}, \ldots, s_{n}\right)$ is a monic polynomial of a degree $k^{n-1}$ with respect to each variable $s_{l}(l=1,2, \ldots, n)$, we have that $(-1)^{k^{n}} H_{n+1, k^{n}}\left(s_{1}, s_{2}, \ldots, s_{n+1}\right)$ is a monic polynomial of a degree $k^{n}$ with respect to each variable $s_{l}(l=1,2, \ldots, n+1)$. It follows from $(2.14)$ to $(2.16)$ that $H_{n+1, j}\left(j=0,1, \ldots, k^{n}\right)$ satisfy (2) in Lemma 2.2. This completes the proof.

Since every integer larger than 1 can be written as a product of one or more primes, we arrive at

Proposition 2.3. Let $k$ and $n$ be two positive integers. Then there exists a homogeneous polynomial $P\left(t_{1}, t_{2}, \ldots, t_{n+1}\right)$ with real coefficients satisfying

(1) the degree of $P$ with respect to each variable is the same;

(2) $P$ is a monic polynomial with respect to $t_{n+1}$;

(3) if $\gamma, t_{1}, t_{2}, \ldots, t_{n} \in \mathbb{R}$ satisfy $\gamma=\sum_{j=1}^{n} t_{j}$, then

$$
P\left(t_{1}^{k}, t_{2}^{k}, \ldots, t_{n}^{k}, \gamma^{k}\right)=0 .
$$

Remark 2.4. Proposition 2.3 shows that there exists a real homogeneous polynomial $P$ such that any zero of

$$
p\left(t_{1}, t_{2}, \ldots, t_{n}, \gamma\right) \equiv \sum_{i=1}^{n} \sqrt[k]{t_{i}}-\gamma
$$

is a zero of

$$
P\left(t_{1}, t_{2}, \ldots, t_{n}, \gamma^{k}\right) \text {. }
$$

Proposition 2.5. Let $G$ be a nonempty open set of $\mathbb{R}^{3}$. If $p \in \mathcal{P}_{\mathbb{Q}_{+}}(G)$ and $\operatorname{deg} p>0$, then for any nonempty open set $G_{0} \subset G$, there exists $x_{0} \in G_{0}$ such that $p\left(x_{0}\right) \neq 0$.

Proof. We see from the definition of $\mathcal{P}_{\mathbb{Q}_{+}}(G)$ that

$$
p=\sum_{i=1}^{n} a_{\alpha^{(i)}} x^{\alpha^{(i)}},
$$

where $\alpha^{(i)} \in \mathbb{Q}_{+}^{3}, 0 \neq a_{\alpha^{(i)}} \in \mathbb{R}$, and $\alpha^{(n)}$ is the max index. Hence we can choose a positive integer $k$ such that all components of $k \alpha^{(i)}(i=1,2, \ldots, n)$ are integers.

Assume that $p=0$ in $G_{0}$. Then there exists a real homogeneous polynomial $P\left(t_{1}, t_{2}, \ldots, t_{n}\right)$ satisfying $(2)$ of Proposition 2.3 and

$$
P\left(x^{k \alpha^{(1)}}, x^{k \alpha^{(2)}}, \ldots, x^{k \alpha^{(n)}}\right)=0, \quad \text { in } G_{0} .
$$

Set $Q=P\left(x^{k \alpha^{(1)}}, x^{k \alpha^{(2)}}, \ldots, x^{k \alpha^{(n)}}\right)$. Then $Q$ is a polynomial with a positive degree which is a contradiction to $Q=0$. This completes the proof.

Lemma 2.6. Let $n \geq 2$ be a positive integer, $G$ and $G_{0}$ be nonempty open subsets of $\mathbb{R}^{3}$ with $G_{0} \subset G$. Let $p_{j} \in \mathcal{P}_{\mathbb{Q}_{+}}^{1 / k}(G)$ for some positive integer $k$ and $q_{j} \in \mathcal{P}_{\mathbb{Q}_{+}}(G)(j=1,2, \ldots, n)$. If $q_{j}>0(j=1,2, \ldots, n)$ in $G_{0}$ with $q_{j_{0}} \neq 1$ in $G_{0}$ for some $j_{0}$ and

$$
\operatorname{deg} p_{j_{0}}>\operatorname{deg} p_{j} \quad \forall j \in\{1,2, \ldots, n\} \backslash\left\{j_{0}\right\},
$$

then there exists $x_{0} \in G_{0}$ such that

$$
\sum_{j=1}^{n} p_{j}\left(x_{0}\right) \ln q_{j}\left(x_{0}\right) \neq 0 .
$$

The proof of Lemma 2.6 is provided in Appendix B. 


\section{BeHAVIOR OF EIGENFUNCTION}

In this section, we investigate the non-polynomial behavior of eigenfunctions of (1.1), which will be applied to analyze the convergence of their adaptive finite element approximations. We see from $[17,34]$ that the eigenfunctions of (1.1) vary rapidly around nuclei, which indeed results in applying adaptive finite element computations.

We first recall the unique continuation property.

Definition 3.1. Equation (1.1) has a unique continuation property if every solution in $H_{\mathrm{loc}}^{2}(\Omega)$ that vanishes on an open set of $\Omega$ vanishes identically.

To look into if (1.1) has a unique continuation property, we may apply the following conclusion, which can be found in [33].

Lemma 3.2. Assume that $u \in H_{l o c}^{2}(\Omega)$ and $\mathcal{V} \in L_{\text {loc }}^{3 / 2}(\Omega)$ such that $|\Delta u| \leq \mathcal{V}|u|$ a.e.. If $u$ vanishes on an open set of $\Omega$, then $u$ is identically zero on $\Omega$.

Theorem 3.3. If $V \in L^{2}(\Omega)$ and $\mathcal{N}_{1}(t) \in \mathscr{P}\left(s,\left(c_{1}, c_{2}\right)\right)$ with $s \in[0,3 / 2]$, then (1.1) has a unique continuation property.

Proof. It follows from a standard elliptic regularity argument that $\phi_{i} \in H^{2}(\Omega)$ (see, e.g., [17,34] and Sect. 4.3.1 of [23]), which together with Sobolev imbedding theorem leads to $\phi_{i} \in C(\bar{\Omega})(i=1,2, \ldots, N)$.

Note that Young's inequality and Sobolev imbedding theorem imply

$$
\left\|\mathcal{N}_{2}(\rho)\right\|_{0, \infty, \Omega} \leq C\|\rho\|_{0, \Omega} \leq C \sum_{i=1}^{N}\left\|\phi_{i}\right\|_{0,4, \Omega}^{2} \leq C\|\Phi\|_{1, \Omega}^{2}<\infty .
$$

We have that $\left|\Delta \phi_{i}\right|=\mathcal{V}_{i}\left|\phi_{i}\right|$ and $\mathcal{V}_{i} \in L_{\text {loc }}^{3 / 2}(\Omega)$, where $\mathcal{V}_{i}=\left|V+\mathcal{N}(\rho)-\lambda_{i}\right| / \kappa(i=1,2, \ldots, N)$. Thus we arrive at the conclusion from Lemma 3.2.

Remark 3.4. We may see from the proof of Theorem 3.3 that if $V \in L^{2}(\Omega)$ is replaced by $V \in L_{\text {loc }}^{3 / 2}(\Omega)$ and any solution of $(1.1)$ is in $H^{2}(\Omega)$, then (1.1) has a unique continuation property.

Theorem 3.5. Let $\Phi=\left(\phi_{1}, \phi_{2}, \ldots, \phi_{N}\right)$ be a solution of (1.1). Assume that $V$ and $\mathcal{N}$ are defined by (2.1) and (2.2)-(2.4) with $\alpha=0$, respectively. If $V$ is a non-constant function and

$$
\operatorname{deg} p_{1}-\operatorname{deg} h_{1}>\max \left\{0, \max _{1 \leq j \leq M}\left(\operatorname{deg} f_{j}-\operatorname{deg} g_{j}\right) / 2, \max _{2 \leq i \leq K}\left(\operatorname{deg} p_{i}-\operatorname{deg} h_{i}\right)\right\}
$$

then for any nonempty open set $G \subset \Omega$, there exists an eigenfunction $\phi_{j}(j \in\{1,2, \ldots, N\})$ being not a non-zero polynomial on $G$. If in addition, $V \in L^{2}(\Omega)$ and $\mathcal{N}_{1}(t) \in \mathscr{P}\left(s,\left(c_{1}, c_{2}\right)\right)$ with $s \in[0,3 / 2]$, then for any nonempty open set $G \subset \Omega$, there exists an eigenfunction $\phi_{j}(j \in\{1,2, \ldots, N\})$ being not a polynomial on $G$.

Proof. Assume that all eigenfunctions $\left\{\phi_{1}, \phi_{2}, \ldots, \phi_{N}\right\}$ are polynomials on the nonempty open set $G$ : $\phi_{j} \in$ $\mathcal{P}_{\ell}(G)(j=1,2, \ldots, N)$ for some positive integer $\ell$. Without loss of generality, let $\operatorname{deg} \phi_{1} \geq \max _{2 \leq j \leq N} \operatorname{deg} \phi_{j}$. We have $\operatorname{deg} \rho=2 \operatorname{deg} \phi_{1}$ and see from (1.1) that

$$
-\kappa \Delta \phi_{1}-\sum_{j=1}^{M} \frac{f_{j}}{g_{j}} \phi_{1}+\sum_{i=1}^{K} \frac{p_{i}(\rho)}{h_{i}(\rho)} \phi_{1} \ln q_{i}(\rho)=\lambda_{1} \phi_{1}, \text { in } G .
$$


If $\operatorname{deg} \phi_{1}>0$, then we see from (3.1) that

$$
\begin{array}{ll}
\left(\operatorname{deg} p_{1}-\operatorname{deg} h_{1}\right) \operatorname{deg} \rho+\operatorname{deg} \phi_{1}>\operatorname{deg} \Delta \phi_{1}, & \\
\left(\operatorname{deg} p_{1}-\operatorname{deg} h_{1}\right) \operatorname{deg} \rho+\operatorname{deg} \phi_{1}>\operatorname{deg}\left(\frac{f_{j}}{g_{j}} \phi_{1}\right), & j=1,2, \ldots, N, \\
\left(\operatorname{deg} p_{1}-\operatorname{deg} h_{1}\right) \operatorname{deg} \rho+\operatorname{deg} \phi_{1}>\left(\operatorname{deg} p_{i}-\operatorname{deg} h_{i}\right) \operatorname{deg} \rho+\operatorname{deg} \phi_{1}, & i=2,3, \ldots, K, \\
\left(\operatorname{deg} p_{1}-\operatorname{deg} h_{1}\right) \operatorname{deg} \rho+\operatorname{deg} \phi_{1}>\operatorname{deg} \phi_{1} . &
\end{array}
$$

Since $q_{i}$ are polynomials implying $q_{i}(\rho) \in \mathcal{P}_{\mathbb{Q}_{+}}(\Omega)(i=1,2, \ldots, K)$, we obtain from Lemma 2.6 that

$$
-\kappa \Delta \phi_{1}\left(x_{0}\right)-\sum_{j=1}^{M} \frac{f_{j}\left(x_{0}\right)}{g_{j}\left(x_{0}\right)} \phi_{1}\left(x_{0}\right)+\sum_{i=1}^{K} \frac{p_{i}(\rho)\left(x_{0}\right)}{h_{i}(\rho)\left(x_{0}\right)} \phi_{1}\left(x_{0}\right) \ln q_{i}(\rho)\left(x_{0}\right) \neq \lambda_{1} \phi_{1}\left(x_{0}\right)
$$

for some $x_{0} \in G$, which is a contradiction to (3.2). Thus we arrive at that $\operatorname{deg} \phi_{1}=0$ on $G$. Since $\operatorname{deg} \phi_{1} \geq$ $\max _{2 \leq j \leq N} \operatorname{deg} \phi_{j}$, we have that $\phi_{j}=c_{j}(j=1,2, \ldots, N)$ are constants on $G$. If $c_{j} \neq 0$ for all $j \in\{1,2, \ldots, N\}$, then

$$
\sum_{j=1}^{M} \frac{f_{j}}{g_{j}}=\sum_{i=1}^{K} \frac{p_{i}(\rho)}{h_{i}(\rho)} \ln q_{i}(\rho)-\lambda_{1}, \quad \text { in } G,
$$

with the constant $\rho=\sum_{j=1}^{N} c_{j}^{2}$, which is impossible. Hence $c_{j}=0$ for some $j \in\{1,2, \ldots, N\}$.

If in addition, $V \in L^{2}(\Omega)$ and $\mathcal{N}_{1}(t) \in \mathscr{P}\left(s,\left(c_{1}, c_{2}\right)\right)$ with $s \in[0,3 / 2]$, then Theorem 3.3 implies that $\phi_{j}=0$ in $\Omega$ for some $j \in\{1,2, \ldots, N\}$, which is a contradiction to $\int_{\Omega} \phi_{j}^{2}=1$. This completes the proof.

Remark 3.6. We see from the above conclusion that the eigenfunction cannot be a polynomial on any open set when $N=1$.

Remark 3.7. Note that Theorem 3.5 may be also true even if

$$
\operatorname{deg} p_{1}-\operatorname{deg} h_{1}=\max _{1 \leq j \leq M}\left(\operatorname{deg} f_{j}-\operatorname{deg} g_{j}\right) / 2 .
$$

For instance, no eigenfunction $\phi \in H^{2}(\Omega)$ of GPE $[2,35]$

$$
\left(-\frac{1}{2} \Delta+V+\beta|\phi|^{2}\right) \phi=\lambda \phi
$$

with a harmonic trap potential

$$
V(x)=\gamma_{1} \xi_{1}^{2}+\gamma_{2} \xi_{2}^{2}+\gamma_{3} \xi_{3}^{2}, \gamma_{1}, \gamma_{2}, \gamma_{3}>0
$$

can be a polynomial on any open set $G \subset \Omega$, where $x=\left(\xi_{1}, \xi_{2}, \xi_{3}\right) \in \mathbb{R}^{3}$.

Theorem 3.8. Let $\Phi=\left(\phi_{1}, \phi_{2}, \ldots, \phi_{N}\right)$ be a solution of (1.1). Assume that $V$ and $\mathcal{N}$ are defined by (2.1) and (2.2)-(2.4) with $\alpha \neq 0$, respectively, and $\alpha \Delta V$ is not a positive constant function. If either of the following conditions holds:

(2) $\operatorname{deg} q_{1}=0$ and

$$
\max _{1 \leq i \leq K}\left(\operatorname{deg} p_{i}-\operatorname{deg} h_{i}\right) \leq 1 \text { and } \max _{1 \leq j \leq M}\left(\operatorname{deg} f_{j}-\operatorname{deg} g_{j}\right)<4
$$

$$
\operatorname{deg} p_{1}-\operatorname{deg} h_{1}>\max \left\{2, \max _{1 \leq j \leq M}\left(\operatorname{deg} f_{j}-\operatorname{deg} g_{j}\right) / 2, \max _{2 \leq i \leq K}\left(\operatorname{deg} p_{i}-\operatorname{deg} h_{i}\right)\right\},
$$


then for any nonempty open set $G \subset \Omega$, there exists an eigenfunction $\phi_{j}(j \in\{1,2, \ldots, N\})$ being not a non-zero polynomial on $G$. If in addition, $V \in L^{2}(\Omega)$ and $\mathcal{N}_{1}(t) \in \mathscr{P}\left(s,\left(c_{1}, c_{2}\right)\right)$ with $s \in[0,3 / 2]$, then for any nonempty open set $G \subset \Omega$, there exists an eigenfunction $\phi_{j}(j \in\{1,2, \ldots, N\})$ being not a polynomial on $G$.

Proof. Assume that all eigenfunctions $\left\{\phi_{1}, \phi_{2}, \ldots, \phi_{N}\right\}$ are polynomials on nonempty open set $G: \phi_{j} \in$ $\mathcal{P}_{\ell}(G)(j=1,2, \ldots, N)$ for some positive integer $\ell$. Without loss of generality, let $\operatorname{deg} \phi_{1} \geq \max _{2 \leq j \leq N} \operatorname{deg} \phi_{j}$. Obviously, $\operatorname{deg} \rho=2 \operatorname{deg} \phi_{1}$.

If $\phi_{1}(x) \neq 0$ for any $x \in G$ and $\operatorname{deg} \phi_{1}>0$, then we obtain from (1.1) that

$$
-\kappa \frac{\Delta \phi_{1}}{\phi_{1}}-\sum_{j=1}^{M} \frac{f_{j}}{g_{j}}+\alpha \int_{\Omega} \frac{\rho(y)}{|\cdot-y|} \mathrm{d} y+\sum_{i=1}^{K} \frac{p_{i}(\rho)}{h_{i}(\rho)} \ln q_{i}(\rho)=\lambda_{1}, \quad \text { in } G .
$$

Applying the Laplace operator to both sides yields

$$
-\kappa p_{\phi_{1}}-\sum_{j=1}^{M} f_{j, g}-4 \alpha \pi \rho+\sum_{i=1}^{K} p_{i, h, \rho} \ln q_{i}(\rho)+\sum_{i=1}^{K} p_{i, h, q, \rho}=0, \quad \text { in } G,
$$

where

$$
\begin{aligned}
p_{\phi_{1}} & =\Delta\left(\frac{\Delta \phi_{1}}{\phi_{1}}\right), \\
f_{j, g} & =\Delta\left(\frac{f_{j}}{g_{j}}\right), j=1, \ldots, M, \\
p_{i, h, \rho} & =\Delta\left(\frac{p_{i}(\rho)}{h_{i}(\rho)}\right), i=1, \ldots, K, \\
p_{i, h, q, \rho} & =2 \frac{\nabla\left(p_{i}(\rho) / h_{i}(\rho)\right) \cdot \nabla q_{i}(\rho)}{q_{i}(\rho)}+\frac{p_{i}(\rho)\left(q_{i}(\rho) \Delta q_{i}(\rho)-\left|\nabla q_{i}(\rho)\right|^{2}\right)}{h_{i}(\rho) q_{i}^{2}(\rho)}, i=1, \ldots, K .
\end{aligned}
$$

If $\max _{1 \leq i \leq K}\left(\operatorname{deg} p_{i}-\operatorname{deg} h_{i}\right) \leq 1$ and $\max _{1 \leq j \leq M}\left(\operatorname{deg} f_{j}-\operatorname{deg} g_{j}\right)<4$, then only $4 \alpha \pi \rho$ has the max degree.

If $\operatorname{deg} q_{1}=0$ and (3.3) holds, then $p_{1, h, q, \rho}=0$. Thus only one term, which is one term of $p_{1, h, \rho}$, has the max degree.

Therefore, we get a contradiction to (3.4) from Lemma 2.6. Consequently, $\operatorname{deg} \phi_{1} \geq \max _{2 \leq j \leq N} \operatorname{deg} \phi_{j} \operatorname{leads}$ to that $\phi_{j}=c_{j}(j=1,2, \ldots, N)$ are constants in $G$.

If $c_{j} \neq 0$ for all $j \in\{1,2, \ldots, N\}$, we then derive from (3.4) that

$$
\Delta V=4 \alpha \pi \sum_{i=1}^{N} c_{i}^{2} \quad \forall x \in G,
$$

which is impossible. Hence $c_{j}=0$ for some $j \in\{1,2, \ldots, N\}$.

If in addition, $V \in L^{2}(\Omega)$ and $\mathcal{N}_{1}(t) \in \mathscr{P}\left(s,\left(c_{1}, c_{2}\right)\right)$ with $s \in[0,3 / 2]$, then we complete the proof by using Theorem 3.3.

We may apply Theorem 3.5 or Theorem 3.8 to the typical mathematical models in quantum physics to see the eigenfunction behavior. The following are some examples.

Example 3.9. No eigenfunction of the Schrödinger-Newton equation [18]

$$
\left(-\Delta-\int_{\Omega} \frac{|u(y)|^{2}}{|\cdot-y|} \mathrm{d} y\right) u=\lambda u, \text { in } \mathbb{R}^{3}
$$

can be a polynomial on any open set of $\mathbb{R}^{3}$. 
Example 3.10. No eigenfunction of the Thomas-Fermi-Dirac-von Weizsäcker equation $[7,21]$

$$
\left(-\kappa \Delta-\sum_{j=1}^{M} \frac{Z_{j}}{\left|\cdot-r_{j}\right|}+\int_{\Omega} \frac{|u(y)|^{2}}{|\cdot-y|} \mathrm{d} y+\beta_{1} u^{2 \nu-2}-\beta_{2} u^{2 / 3}\right) u=\lambda u
$$

can be a polynomial locally for a rational number $\nu$ in $[1,2]$, where $\beta_{1}$ and $\beta_{2}$ are constants.

Example 3.11. The Kohn-Sham equation of a system consisting of $M$ nuclei of charges $\left\{Z_{1}, Z_{2}, \ldots, Z_{M}\right\}$ located at the positions $\left\{r_{1}, r_{2}, \ldots, r_{M}\right\}$ and $N$ electrons is as follows:

$$
\left\{\begin{array}{c}
\left(-\frac{1}{2} \Delta+V_{\mathrm{ext}}+\int_{\Omega} \frac{\rho(y)}{|\cdot-y|} \mathrm{d} y+V_{\mathrm{xc}}(\rho)\right) \phi_{i}=\lambda_{i} \phi_{i}, \text { in } \Omega, i=1,2, \ldots, N, \\
\int_{\Omega} \phi_{i} \phi_{j}=\delta_{i j}
\end{array}\right.
$$

where $V_{\text {ext }}=-\sum_{k=1}^{M} \frac{Z_{k}}{\left|--r_{k}\right|}$ is the associated external potential, $\rho=\sum_{i=1}^{N}\left|\phi_{i}\right|^{2}$ is the electronic density, and $V_{\mathrm{xc}}(\rho)$ is the exchange-correlation potential such as the $X_{\alpha}$ exchange-correction potential [30]

$$
V_{\mathrm{xc}}(\rho)=\frac{3}{2} \alpha\left(\frac{3}{\pi} \rho\right)^{1 / 3}
$$

with $\alpha \in[2 / 3,1]$ or the Perdew-Zunger type local-density approximations (LDA) potential [26]:

$$
V_{\mathrm{xc}}^{\mathrm{LDA}}(\rho)= \begin{cases}-\frac{0.1423+0.0633 r_{s}+0.1748 \sqrt{r_{s}}}{\left(1+1.0529 \sqrt{r_{s}}+0.3334 r_{s}\right)^{2}}-\left(\frac{9}{4 \pi^{2}}\right)^{\frac{1}{3}} \frac{1}{r_{s}}, & \text { if } r_{s} \geq 1, \\ 0.0311 \ln r_{s}-0.0584+0.0013 r_{s} \ln r_{s}-0.0084 r_{s}-\left(\frac{9}{4 \pi^{2}}\right)^{\frac{1}{3}} \frac{1}{r_{s}}, & \text { if } r_{s}<1\end{cases}
$$

with $r_{s}=\left(\frac{3}{4 \pi \rho}\right)^{1 / 3}$. It is clear that $V_{\text {ext }}$ can be rewritten as the form (2.1). And (3.6) and (3.7) can be rewritten as the form (2.3).

We see that if the exchange-correction potential is chosen as either (3.6) or (3.7), then for any nonempty open set $G \subset \Omega$, there exists an eigenfunction $\phi_{j}(j \in\{1,2, \ldots, N\})$ being not a polynomial on $G$.

In fact, the same conclusion is true for the Vosko-Wilk-Nusair type LDA potential [32]

$$
\begin{aligned}
V_{\mathrm{xc}}(\rho)= & \frac{A}{2}\left\{\ln \frac{t^{2}}{X(t)}+\frac{2 b}{Q} \tan ^{-1} \frac{Q}{2 t+b}-\frac{b t_{0}}{X\left(t_{0}\right)}\left(\ln \frac{\left(t-t_{0}\right)^{2}}{X(t)}\right.\right. \\
& \left.\left.+\frac{2\left(b+2 t_{0}\right)}{Q} \tan ^{-1} \frac{Q}{2 t+b}\right)\right\}
\end{aligned}
$$

where $r_{s}=\left(\frac{3}{4 \pi \rho}\right)^{1 / 3}, t=r_{s}^{1 / 2}, X(t)=t^{2}+b t+c, Q=\left(4 c-b^{2}\right)^{1 / 2}, A=0.0621814, t_{0}=-0.409286, b=13.0720$, and $c=42.7198$.

Indeed, we conjecture that no eigenfunction of (1.1) can be a polynomial on any open set in $\mathbb{R}^{3}$ when $N>1$. Unfortunately, it is still open whether it is true or not. 


\section{Adaptive approximations}

In this section, we apply the behavior of the eigenfunctions to investigate the convergence of adaptive finite element approximations of (1.1). We assume that

(i) $V \in L^{2}(\Omega)$;

(ii) $\mathcal{E} \in \mathscr{P}\left(3,\left(c_{1}, c_{2}\right)\right)$ with $c_{1} \geq 0$ or $\mathscr{P}\left(4 / 3,\left(c_{1}, c_{2}\right)\right)$;

(iii) $\mathcal{N}_{1} \in \mathscr{P}\left(s_{1},\left(c_{1}, c_{2}\right)\right)$ for some $s_{1} \in[0,2)$ and $t \mathcal{N}^{\prime}(t) \in \mathscr{P}\left(s_{2},\left(\tilde{c}_{1}, \tilde{c}_{2}\right)\right)$ for some $s_{2} \in[0,2)$.

Let

$$
\Omega^{+}=\bigcup_{T \in \mathcal{T}^{+}} T
$$

where

$$
\mathcal{T}^{+}=\bigcup_{k \geq 0} \bigcap_{m \geq k} \mathcal{T}_{m}
$$

In our analysis, we need Lemma 4.3 in [14], which is stated as follows:

Lemma 4.1. The set $\Omega^{+}$is empty if and only if $\lim _{k \rightarrow \infty}\left\|h_{k}\right\|_{0, \infty, \Omega}=0$.

Under the assumptions (i)-(iii), we observe from Theorem 3.5 in [10] and Theorem 4.2 in [7] that approximations $\Theta_{k}$ produced by Algorithm 1 converge to a solution of (1.1) for any initial mesh and the solution becomes a ground state if the initial mesh size is sufficiently small so that $\Theta_{0}$ is sufficiently near to $\Theta$. Indeed, based on the eigenfunction behavior, we are able to prove that $\Theta_{k}$ produced by Algorithm 1 converge to a ground state of (1.1) starting from any initial mesh under some assumptions.

Using the similar argument to the proof of Lemma 6.2 in [14], we have

Lemma 4.2. Let mesh size functions $\left\{h_{k}\right\}_{k \in \mathbb{N}}$ and ground state solutions $\left\{\Theta_{k}=\left(\Lambda_{k}, \Phi_{k}\right)\right\}_{k \in \mathbb{N}}$ be produced by Algorithm 1. If for any nonempty open set $G \subset \Omega$, there exists an eigenfunction of (1.1) being not a polynomial on $G$, then $\left\|h_{k}\right\|_{0, \infty, \Omega} \rightarrow 0$ as $k \rightarrow \infty$.

Proof. If $\left\|h_{k}\right\|_{0, \infty, \Omega}$ does not tend to zero, then we derive from Lemma 4.1 that $\Omega^{+}$is not empty. Thus there exists $T \in \mathcal{T}^{+}$and $k_{0} \in \mathbb{N}$ such that $T \in \mathcal{T}_{k}$ for all $k \geq k_{0}$.

Without loss of generality, we assume that $\phi_{1}$ is not a polynomial on the nonempty open set $T$. We obtain from the proof of Theorem 3.5 in [10] that there exists a subsequence $\Phi_{k_{m}}$ and some solution $\Phi$ of (1.1) such that $\Phi_{k_{m}} \rightarrow \Phi$ in $\mathcal{H}$. Thus we have that $\phi_{1, k_{m}} \rightarrow \phi_{1}$ in $H_{0}^{1}(\Omega)$ as $m \rightarrow \infty$, which derives

$$
\lim _{m \rightarrow \infty}\left\|\phi_{1, k_{m}}-\phi_{1}\right\|_{0, T}=0 \text {. }
$$

Combining (4.1), $\left.\phi_{1, k}\right|_{T} \in \mathcal{P}_{n}(T)$ for some integer $n$, and that $\mathcal{P}_{n}(T)$ is a finite dimensional space, we obtain that $\phi_{1} \in \mathcal{P}_{n}(T)$, which contradicts to that $\phi_{1}$ is not a polynomial on $T$. This completes the proof.

Let the distance between sets $X, Y \subset \mathbb{R}^{N \times N} \times \mathcal{H}$ be defined by

$$
d_{\mathcal{H}}(X, Y)=\sup _{(\Lambda, \Phi) \in X} \inf _{(\Sigma, \Psi) \in Y}\left(|\Lambda-\Sigma|+\|\Phi-\Psi\|_{1, \Omega}\right),
$$

where $|\cdot|$ is the Frobenius norm in $\mathbb{R}^{N \times N}$.

To carry out the convergence analysis, the gap-condition

$$
\min _{\Psi \in \mathcal{Q}} E(\Psi)<\inf _{(M, \Psi) \in \mathcal{W} \backslash \Theta} E(\Psi)
$$

is assumed in $[7,10]$. Consequently, we have

$$
\min _{\Psi_{h} \in V_{h} \cap \mathcal{Q}} E\left(\Psi_{h}\right)<\inf _{(M, \Psi) \in \mathcal{W} \backslash \Theta} E(\Psi)
$$


when $h$ is small enough. In order to ensure (4.3), we observe that the initial mesh size $h$ is assumed to be sufficiently small in $[7,10]$ (see [10], Thm. 3.5 and [7], Thm. 4.2). On the one hand, combining Theorems 3.5, 3.8, and Lemma 4.2 , we indeed have that the mesh size $h_{k}$ tends to zero under the assumption in Theorem 3.5 or that in Theorem 3.8, which shows that the mesh size $h_{k}$ will be sufficiently small after finite iteration steps and hence (4.3) holds provided $k \gg 1$. Namely, we have

$$
E\left(\Phi_{k}\right)<\inf _{(M, \Psi) \in \mathcal{W} \backslash \Theta} E(\Psi)
$$

provided $k \gg 1$. On the other hand, we see that for any subsequence $\left\{\left(\Lambda_{k_{m}}, \Phi_{k_{m}}\right)\right\}_{m \in \mathbb{N}}$, there exists a convergent subsequence $\left\{\left(\Lambda_{k_{m_{j}}}, \Phi_{k_{m_{j}}}\right)\right\}_{j \in \mathbb{N}}$ and $\left(\Lambda_{\infty}, \Phi_{\infty}\right) \in \mathcal{W}$ such that $\left(\Lambda_{k_{m_{j}}}, \Phi_{k_{m_{j}}}\right) \rightarrow\left(\Lambda_{\infty}, \Phi_{\infty}\right)$ (see the proofs of Thm. 3.5 in [10] and Thm. 4.2 in [7] for more details). Hence we have $\left(\Lambda_{\infty}, \Phi_{\infty}\right) \in \Theta$ and arrive at

Theorem 4.3. Let $\left\{\Theta_{k}\right\}_{k \in \mathbb{N}}$ be the sequence generated by Algorithm 1. If the gap-condition (4.2) and the assumption in Theorem 3.5 or that in Theorem 3.8 are satisfied, then

$$
\begin{gathered}
\lim _{k \rightarrow \infty} E_{k}=\min _{\Psi \in \mathcal{Q}} E(\Psi), \\
\lim _{k \rightarrow \infty} d_{\mathcal{H}}\left(\Theta_{k}, \Theta\right)=0,
\end{gathered}
$$

where $E_{k}=E(\Phi)\left((\Lambda, \Phi) \in \Theta_{k}\right)$.

Remark 4.4. We mention that we do not require the assumption in Theorem 4.3 that the ground state solution and the discrete ground state solution correspond to the lowest $N$ eigenpairs of (2.7) and (2.10), respectively. In practice, however, $\Theta$ and $\Theta_{h}$ are assumed to be the lowest $N$ eigenpairs of (2.7) and (2.10), respectively [22].

As a result, we see from $[8,10]$ that the adaptive finite element method has the asymptotic linear convergence rate and the asymptotic optimal complexity from any initial mesh. More precisely, the adaptive finite element method has the linear convergence rate and the optimal complexity after finite iteration steps.

\section{CONCLUding REMARKS}

In this paper, we have investigated a class of nonlinear eigenvalue problems modeling quantum physics. We have first proved that for any open set $G$, there exists an eigenfunction that cannot be a polynomial on $G$, which may be reviewed as a refinement of the standard unique continuation property. Then applying the nonpolynomial behavior of eigenfunctions, we have shown that adaptive finite element approximations converge to some ground state even if the initial mesh is not fine.

We mention that the same conclusion can be expected for any dimensions larger than 3. For instance, our arguments can be applied to the following linear eigenvalue problem:

$$
-\nabla \cdot(\mathcal{A} \nabla u)+\mathcal{V} u=\lambda \mathcal{B} u, \quad \text { in } \Omega,
$$

where $\Omega \subset \mathbb{R}^{d}$ for some positive integer $d \geq 3$ and $\mathcal{A}$ is a symmetric-matrix-valued function and is uniformly positive definite. We see that (5.1) includes the electronic Schrödinger equation

$$
\left(-\sum_{i=1}^{N} \frac{\hbar^{2}}{2 m_{e}} \nabla_{x_{i}}^{2}-\sum_{i=1}^{N} \sum_{j=1}^{M} \frac{Z_{j} e^{2}}{\left|x_{i}-r_{j}\right|}+\frac{1}{2} \sum_{i, j=1, i \neq j}^{N} \frac{e^{2}}{\left|x_{i}-x_{j}\right|}\right) \phi=E \phi, \quad \text { in } \mathbb{R}^{3 N},
$$

where $\hbar$ is the Planck's constant divided by $2 \pi, m_{e}$ is the mass of the electron, $\left\{x_{i}: i=1, \ldots, N\right\}$ are variables that describe the electron positions, and $e$ is the electronic charge, $\phi$ is the wavefunction, $N$ is the number of electrons, $M$ is the number of atoms, $Z_{j}$ is the atomic number of the $j$-th atom, and $r_{j}$ is the position of the $j$-th atom.

For convenience, we introduce the following assumptions: 
I Entries of $\mathcal{A}$ are continuous and piecewise functions in $\mathcal{P}_{\mathbb{Q}_{+}}(\Omega)$.

II $\mathcal{B}$ is a piecewise function in $\mathcal{P}_{\mathbb{Q}_{+}}(\Omega)$.

III $\mathcal{V}=-\sum_{j=1}^{M} \frac{f_{j}}{g_{j}}$, where $f_{j}, g_{j}(j=1,2, \ldots, M)$ are piecewise functions in $\mathcal{P}_{\mathbb{Q}_{+}}^{\mu}(\Omega)$ for some $\mu \in \mathbb{Q}_{+}$.

IV $\mathcal{V}$ cannot be equal to $\lambda \mathcal{B}$ for any $\lambda \in \mathbb{R}$ in any open subset of $\Omega$.

If Assumptions I-IV hold true and that the entries of $\left.\mathcal{A}\right|_{G},\left.\mathcal{B}\right|_{G}$ belong to $\mathcal{P}_{\mathbb{Q}_{+}}(G)$ and $\left.f_{j}\right|_{G},\left.g_{j}\right|_{G} \in \mathcal{P}_{\mathbb{Q}_{+}}^{\mu}(G)$ for all $j \in\{1,2, \ldots, M\}$ imply that only one among $\left.\operatorname{deg} \mathcal{A}\right|_{G}-2,\left.\operatorname{deg} \mathcal{B}\right|_{G},\left.\operatorname{deg} f_{1}\right|_{G}-\left.\operatorname{deg} g_{1}\right|_{G}, \ldots,\left.\operatorname{deg} f_{M}\right|_{G}-$ $\left.\operatorname{deg} g_{M}\right|_{G}$ equals to

$$
\max \left\{\left.\operatorname{deg} \mathcal{A}\right|_{G}-2,\left.\operatorname{deg} f_{1}\right|_{G}-\left.\operatorname{deg} g_{1}\right|_{G}, \ldots,\left.\operatorname{deg} f_{M}\right|_{G}-\left.\operatorname{deg} g_{M}\right|_{G},\left.\operatorname{deg} \mathcal{B}\right|_{G}\right\}
$$

when $G \subset \Omega$ is an open subset, then no eigenfunction of (5.1) can be a non-zero polynomial on $G$. If in addition, (5.1) has a unique continuation property (see, e.g., $[29,33]$ ), then any $H_{\text {loc }}^{2}$-eigenfunction of $(5.1)$ cannot be a polynomial on any open subset of $\Omega$. Since (5.2) satisfies Assumptions I-IV, in particular, we obtain the more sophisticate conclusion than that in the existing literature (see, e.g., [28]).

Note that the so-called Non-Degeneracy Assumption of a linear case of

$$
-\nabla \cdot(\mathcal{A} \nabla u)=\lambda \mathcal{B} u
$$

has been introduced in [14], which is a special case of (5.1) when $\mathcal{V}=0$, the entries of $\mathcal{A}$ are continuous and piecewise linear, and $\mathcal{B}$ is piecewise constant, with which together the convergence of an adaptive finite element method from any initial mesh for (5.3) is then derived.

\section{Appendix A.}

In this appendix, we provide a proof of Lemma 2.1, whose idea is inspired by Appendixes A and B of [27].

Proof. Let $\gamma=a_{1} t+a_{2} t^{2}+\ldots+a_{k-1} t^{k-1}$ with $\gamma, a_{1}, a_{2}, \ldots, a_{k-1}, t \in \mathbb{R}$. For convenience, we may view $\gamma$ as a polynomial $\gamma(t)$ with respect to $t$. Let $z \neq 1$ be a $k$ th root of 1 . We have

$$
\sum_{j=0}^{k-1} z^{j}=0
$$

and

$$
\left\{z^{m j}: j=1,2, \ldots, k-1\right\}=\left\{z^{j}: j=1,2, \ldots, k-1\right\}
$$

for any positive integer $m$ that is not divisible by $k$. Let

$$
P_{t}(y)=\prod_{m=0}^{k-1}\left(y-\gamma\left(z^{m} t\right)\right), \quad y \in \mathbb{R},
$$

we obtain that $P_{t}(\gamma)=0$ since $z^{0}=1$. We claim that $P_{t}(\gamma)=0$ yields the conclusion.

Indeed, it follows from (A.3) that $P_{t}(y)$ can be rewritten as

$$
P_{t}(y)=y^{k}+\sum_{j=1}^{k} q_{j}\left(a_{1}, a_{2}, \ldots, a_{k-1}, t\right) y^{k-j},
$$

where

$$
q_{j}\left(t_{1}, t_{2}, \ldots, t_{k}\right)=\sum_{\ell=j}^{j(k-1)} f_{j, \ell}\left(t_{1}, t_{2}, \ldots, t_{k-1}\right) t_{k}^{\ell}
$$


for all $t_{1}, t_{2}, \ldots, t_{k} \in \mathbb{R}$ and $f_{j, \ell}$ is either a zero polynomial or a homogeneous polynomial of a degree $j$ for any $\ell=j, j+1, \ldots, j(k-1)$. We see from (A.3) that $\overline{P_{t}(y)}=P_{t}(y)$. Thus $q_{j}(j=1,2, \ldots, k)$ are real polynomials. We obtain from (A.3) to (A.5) that

$$
\begin{aligned}
P_{t}(y) & =\frac{1}{k} \sum_{m=0}^{k-1} P_{z^{m} t}(y)=y^{k}+\sum_{j=1}^{k}\left(\frac{1}{k} \sum_{m=0}^{k-1} q_{j}\left(a_{1}, a_{2}, \ldots, a_{k-1}, z^{m} t\right)\right) y^{k-j} \\
& =y^{k}+\sum_{j=1}^{k}\left(\frac{1}{k} \sum_{\ell=j}^{j(k-1)} f_{j, \ell}\left(a_{1}, a_{2} \ldots, a_{k-1}\right) t^{\ell} \sum_{m=0}^{k-1} z^{\ell m}\right) y^{k-j}
\end{aligned}
$$

which together with (A.1) and (A.2) leads to

$$
P_{t}(y)=y^{k}+\sum_{j=2}^{k}\left(\sum_{m=1}^{j-1} f_{j, m k}\left(a_{1}, a_{2}, \ldots, a_{k-1}\right) t^{m k}\right) y^{k-j} .
$$

Comparing (A.4) with (A.6), we arrive at

$$
\begin{aligned}
& q_{1}\left(t_{1}, t_{2}, \ldots, t_{k}\right)=0 \\
& q_{j}\left(t_{1}, t_{2}, \ldots, t_{k}\right)=\sum_{m=1}^{j-1} f_{j, m k}\left(t_{1}, t_{2}, \ldots, t_{k-1}\right) t_{k}^{m k}, j=2, \ldots, k
\end{aligned}
$$

for all $t_{1}, t_{2}, \ldots, t_{k} \in \mathbb{R}$. Let

$$
p_{j}\left(t_{1}, t_{2}, \ldots, t_{k}\right)=\sum_{m=1}^{j-1} f_{j, m k}\left(t_{1}, t_{2}, \ldots, t_{k-1}\right) t_{k}^{m}, j=2, \ldots, k,
$$

then we have

$$
q_{j}\left(t_{1}, t_{2}, \ldots, t_{k}\right)=p_{j}\left(t_{1}, t_{2}, \ldots, t_{k-1}, t_{k}^{k}\right), j=2,3, \ldots, k .
$$

Thus we complete the proof by using $P_{t}(\gamma)=0$, (A.7), (A.8), and that $f_{j, \ell}$ is either a zero polynomial or homogeneous of a degree $j$ for any $\ell$.

\section{Appendix B.}

In this appendix, we provide a proof of Lemma 2.6.

Proof. Without loss of generality, we divide $n$ into two parts: $n=n_{1}+n_{2}$, such that $\operatorname{deg} q_{j}=0$ for $j \in\left\{1, \ldots, n_{1}\right\}$ and $\operatorname{deg} q_{j}>0$ for $j \in\left\{n_{1}+1, \ldots, n_{1}+n_{2}\right\}$.

We prove the conclusion by induction on $n_{2}$.

(1) For $n_{2}=0$, we prove the conclusion by contradiction again. Assume that

$$
\sum_{j=1}^{n_{1}} p_{j} \ln q_{j}=0, \quad \text { in } G_{0}
$$

where $\ln q_{j}$ are constants and $\ln q_{j_{0}}(x) \neq 0$ for any $x \in G_{0}$.

For convenience, we assume $j_{0}=1$. Let $P\left(t_{1}, t_{2}, \ldots, t_{n_{1}}\right)$ be a real homogeneous polynomial satisfying $(2)$ of Proposition 2.3 and

$$
P\left(p_{2}^{k}, \ldots, p_{n_{1}}^{k}, p_{1}^{k}\right)=0, \quad \text { in } G_{0} .
$$


Set $Q=P\left(p_{2}^{k}, \ldots, p_{n}^{k}, p_{1}^{k}\right)$. We get from $p_{j} \in \mathcal{P}_{\mathbb{Q}_{+}}^{1 / k}\left(j=1,2, \ldots, n_{1}\right)$ that $Q \in \mathcal{P}_{\mathbb{Q}_{+}}$. Note that $P$ is a homogeneous polynomial and monic in $t_{n_{1}}$, we obtain from the definition of $Q$ and $\operatorname{deg} p_{1}>\max _{2 \leq j \leq n_{1}} \operatorname{deg} p_{j}$ that $\operatorname{deg} Q>0$. Therefore Proposition 2.5 leads to a contradiction to $Q=0$ in $G_{0}$. Thus Lemma 2.6 is true for $n_{2}=0$.

(2) Assume that Lemma 2.6 is true for $n_{2} \geq 0$. We show that Lemma 2.6 is true for $n_{2}+1$. Let $j_{0}=1$ or $j_{0}=n_{1}+1$. It is obvious that the conclusion is true if $p_{n_{1}+1}=0$ in $G_{0}$. If $p_{n_{1}+1} \neq 0$ in $G_{0}$, then we assume that

$$
\sum_{j=1}^{n_{1}} p_{j} \ln q_{j}+\sum_{j=n_{1}+1}^{n_{1}+n_{2}+1} p_{j} \ln q_{j}=0, \quad \text { in } G_{0},
$$

which leads to

$$
\sum_{j=1}^{n_{1}} \frac{p_{j}}{p_{n_{1}+1}} \ln q_{j}+\ln q_{n_{1}+1}+\sum_{j=n_{1}+2}^{n_{1}+n_{2}+1} \frac{p_{j}}{p_{n_{1}+1}} \ln q_{j}=0, \quad \text { in } \tilde{G}
$$

for some nonempty open subset $\tilde{G} \subset G_{0}$, where $q_{j}\left(j=1, \ldots, n_{1}\right)$ are constants. Applying $\partial_{i}$ to (B.1) $(i=1,2,3)$, we obtain

$$
\sum_{j=1}^{n_{1}} \frac{\tilde{p}_{j, i}}{p_{n_{1}+1}^{2}} \ln q_{j}+\sum_{j=n_{1}+2}^{n_{1}+n_{2}+1} \frac{p_{j} \partial_{i} q_{j}}{p_{n_{1}+1} q_{j}}+\frac{\partial_{i} q_{n_{1}+1}}{q_{n_{1}+1}}+\sum_{j=n_{1}+2}^{n_{1}+n_{2}+1} \frac{\tilde{p}_{j, i}}{p_{n_{1}+1}^{2}} \ln q_{j}=0, \quad \text { in } \tilde{G},
$$

where $\tilde{p}_{j, i}=p_{n_{1}+1} \partial_{i} p_{j}-p_{j} \partial_{i} p_{n_{1}+1}, j=1, \ldots, n_{1}+n_{2}+1, i=1,2,3$. It is easy to see that $p_{n_{1}+1}^{k} p_{j}^{k} \xi_{i} \tilde{p}_{j, i} \in$ $\mathcal{P}_{\mathbb{Q}_{+}}^{1 / k}$.

If $j_{0}=1$, then there exists $i$ such that $\operatorname{deg} \tilde{p}_{1, i}=\operatorname{deg} p_{1}+\operatorname{deg} p_{n_{1}+1}-1$. Thus we have

$$
\begin{array}{ll}
\operatorname{deg} \tilde{p}_{1, i}-\operatorname{deg} p_{n_{1}+1}^{2}>\operatorname{deg} \tilde{p}_{j, i}-\operatorname{deg} p_{n_{1}+1}^{2}, & j=2, \ldots, n_{1}+n_{2}+1, \\
\operatorname{deg} \tilde{p}_{1, i}-\operatorname{deg} p_{n_{1}+1}^{2}>\operatorname{deg}\left(p_{j} \partial_{i} q_{j}\right)-\operatorname{deg}\left(p_{n_{1}+1} q_{j}\right), & j=n_{1}+2, \ldots, n_{1}+n_{2}+1, \\
\operatorname{deg} \tilde{p}_{1, i}-\operatorname{deg} p_{n_{1}+1}^{2}>\operatorname{deg} \partial_{i} q_{n_{1}+1}-\operatorname{deg} q_{n_{1}+1} . &
\end{array}
$$

If $j_{0}=n_{1}+1$, then we pick up $i$ satisfying $\operatorname{deg} \partial_{i} q_{n_{1}+1} \neq-\infty$. It follows that

$$
\operatorname{deg} \partial_{i} q_{n_{1}+1}-\operatorname{deg} q_{n_{1}+1}=-1 .
$$

Consequently,

$$
\begin{array}{llrl}
\operatorname{deg} \partial_{i} q_{n_{1}+1}-\operatorname{deg} q_{n_{1}+1}>\operatorname{deg} \tilde{p}_{j, i}-\operatorname{deg} p_{n_{1}+1}^{2}, & j & =1, \ldots, n_{1}+n_{2}+1, \\
\operatorname{deg} \partial_{i} q_{n_{1}+1}-\operatorname{deg} q_{n_{1}+1}>\operatorname{deg}\left(p_{j} \partial_{i} q_{j}\right)-\operatorname{deg}\left(p_{n_{1}+1} q_{j}\right), & & j=n_{1}+2, \ldots, n_{1}+n_{2}+1 .
\end{array}
$$

Thus we conclude from the induction hypothesis that Lemma 2.6 is true when $n_{2}$ is replaced by $n_{2}+1$. This completes the proof.

Acknowledgements. The authors would like to thank the referees for their comments and suggestions that improved the presentation of this paper. This work was partially supported by National Key Research and Development of China under grant 2019YFA0709601, the Key Research Program of Frontier Sciences of the Chinese Academy of Sciences under grant QYZDJ-SSW-SYS010, and the National Science Foundation of China under grants 91730302 and 11671389.

\section{REFERENCES}

[1] R.A. Adams, Sobolev Spaces. Academic Press, New York (1975).

[2] W. Bao and Y. Cai, Mathematical theory and numerical methods for Bose-Einstein condensation. Kinet. Relat. Mod. 6 (2013) $1-135$. 
[3] A.D. Becke, Perspective: fifty years of density-functional theory in chemical physics. J. Chem. Phys. 140 (2014) 18A301.

[4] A. Bonito and A. Demlow, Convergence and optimality of higher-order adaptive finite element methods for eigenvalue clusters. SIAM J. Numer. Anal. 54 (2016) 2379-2388.

[5] C. Canuto, Adaptive hp-FEM for eigenvalue computations. Calcolo 56 (2019) 39.

[6] J.M. Cascon, C. Kreuzer, R.H. Nochetto and K.G. Siebert, Quasi-optimal convergence rate for an adaptive finite element method. SIAM J. Numer. Anal. 46 (2008) 2524-2550.

[7] H. Chen, X. Gong, L. He and A. Zhou, Adaptive finite element approximations for a class of nonlinear eigenvalue problems in quantum physics. Adv. Appl. Math. Mech. 3 (2011) 493-518.

[8] H. Chen, L. He and A. Zhou, Finite element approximations of nonlinear eigenvalue problems in quantum physics. Comput. Methods Appl. Mech. Eng. 200 (2011) 1846-1865.

[9] H. Chen, X. Gong, L. He, Z. Yang and A. Zhou, Numerical analysis of finite dimensional approximations of Khon-Sham models. Adv. Comput. Math. 38 (2013) 225-256.

[10] H. Chen, X. Dai, X. Gong, L. He and A. Zhou, Adaptive finite element approximations for Kohn-Sham models. Multiscale Model. Simul. 12 (2014) 1828-1869.

[11] X. Dai, J. Xu and A. Zhou, Convergence and optimal complexity of adaptive finite element eigenvalue computations. Numer. Math. 110 (2008) 313-355.

[12] X. Dai, L. He and A. Zhou, Convergence and quasi-optimal complexity of adaptive finite element computations for multiple eigenvalues. IMA J. Numer. Anal. 35 (2015) 1934-1977.

[13] D. Davydov, T.D. Young and P. Steinmann, On the adaptive finite element analysis of the Kohn-Sham equations: methods, algorithms, and implementation. Int. J. Numerc. Methods Eng. 106 (2016) 863-888.

[14] E.M. Garau, P. Morin and C. Zuppa, Convergence of adaptive finite element methods for eigenvalue problems. Math. Models Methods Appl. Sci. 19 (2009) 721-747.

[15] D. Gallistl, An optimal adaptive FEM for eigenvalue clusters. Numer. Math. 130 (2015) 467-496.

[16] S. Giani and I.G. Graham, A convergent adaptive method for elliptic eigenvalue problems. SIAM J. Numer. Anal. 47 (2009) 1067-1091.

[17] X. Gong, L. Shen, D. Zhang and A. Zhou, Finite element approximations for Schrödinger equations with applications to electronic structure computations. J. Comput. Math. 23 (2008) 310-327.

[18] R. Harrison, I. Moroz and K.P. Tod, A numerical study of the Schrödinger-Newton equations. Nonlinearity 16 (2003) $101-122$.

[19] D. Jerison and C. E. Kenig, Unique continuation and absence of positive eigenvalues for Schrödinger operators. Ann. Math. 121 (1985) 463-494.

[20] C. Le Bris, ed., Handbook of Numerical Analysis. In: Vol. X of Special issue: Computational Chemistry, North-Holland (2003).

[21] E.H. Lieb, Thomas-Fermi and related theories of atoms and molecules. Rev. Mod. Phys. 53 (1981) 603-641.

[22] R.M. Martin, Electronic Structure: Basic Theory and Practical Method. Cambridge University Press, Cambridge (2004).

[23] V. Maz'ya and J. Rossmann, Elliptic Equations in Polyhedral Domains. American Mathematical Society, Providence, RI (2010).

[24] P. Motamarri, M.R. Nowak, K. Leiter, J. Knap and V. Gavini, Higher-order adaptive finite-element methods for Kohn-Sham density functional theory. J. Comput. Phys. 253 (2013) 308-343.

[25] R. Penrose, On gravity's role in quantum state reduction. Gen. Rel. Grav. 28 (1996) 581-600.

[26] J.P. Perdew and A. Zunger, Self-interaction correction to density-functional approximations for many-electron systems. Phys. Rev. B 23 (1981) 5048-5079.

[27] P. Pesic, Abel's Proof: An Essay on the Sources and Meaning of Mathematical Unsolvability. MIT Press, Cambridge etc (2004).

[28] M. Reed and B. Simon, Methods of Modern Mathematical Physics-IV: Analysis of Operators. Academic Press, San Diego (1978).

[29] M. Schechter and B. Simon, Unique continuation for Schrödinger operators with unbounded potentials. J. Math. Anal. Appl. 77 (1980) 482-492.

[30] J.C. Slater, A simplification of the Hartree-Fock method. Phys. Rev. 81 (1951) 385-390.

[31] E. Tsuchida and M. Tsukada, Adaptive finite-element method for electronic-structure calculations. Phys. Rev. B 54 (1996) 7602-7605.

[32] S.H. Vosko, L. Wilk and M. Nusair, Accurate spin-dependent electron liquid correlation energies for local spin density calculations: a critical analysis. Can. J. Phys. 58 (1980) 1200-1211.

[33] H. Wolff, Recent work on sharp estimates in second-order elliptic unique continuation problems. J. Gome. Anal. 3 (1993) 621-650.

[34] X. Zhang and A. Zhou, A singularity-based eigenfunction decomposition for Kohn-Sham equations. Sci. Sin. Math. 59 (2016) 1623-1634.

[35] A. Zhou, An analysis of finite-dimensional approximations for the ground state solution of Bose-Einstein condensates. Nonlinearity 17 (2004) 541-550.

[36] A. Zhou, Hohenberg-Kohn theorem for Coulomb type systems and its generalization. J. Math. Chem. 50 (2012) $2746-2754$. 